\title{
Fatores de risco aos desfechos obstétricos e neonatais de mães adolescentes
}

\author{
Risk factors for obstetric and neonatal outcomes of adolescent mothers
}

Factores de riesgo para los resultados obstétricos y neonatales de las madres adolescentes

\section{Betina Berlitz ${ }^{I}$, Carlise Rigon Dalla Nora ${ }^{I I}$, Rafaela Schaefer ${ }^{I I I}$, Karin Viegas ${ }^{\text {IV }}$ Marilyn Agranonikv, Rosangela Barbianivi}

Resumo: Objetivo: comparar os desfechos obstétricos e neonatais entre mães adolescentes e adultas. Método: estudo transversal desenvolvido com base nos dados do Sistema de Informações de Nascidos Vivos,-comparando variáveis sociodemográficas, obstétricas e neonatais de mães adolescentes e adultas do Rio Grande do Sul, no período de 2010-2016. O teste Qui-quadrado avaliou associação estatística e foi estimada a razão de prevalência. Resultados: houve diferenças significativas entre mães de 10-14 anos e as dos demais estratos: prevalência de raça/cor preta ou parda ( $\mathrm{RP}=1,53$; IC95\% 1,46-1,60), pré-natal inadequado ( $\mathrm{RP}=1,89$; IC95\% 1,84-1,94), prematuridade $(\mathrm{RP}=1,35$; IC95\% 1,27-1,43), baixo peso ao nascer ( $\mathrm{RP}=1,34$; IC95\% 1,25-1,43) e desempenho inferior no Apgar do 1은 minuto (RP=5,68; IC95\% 5,49-5,88). Conclusão: a prevalência de fatores de risco aos desfechos obstétricos e neonatais de adolescentes de 10-14 anos demanda avaliação criteriosa da saúde. A gravidez precoce na adolescência requer o acionamento de equipes interdisciplinares, redes familiares, socioassistenciais e de proteção sociojurídica. Descritores: Gravidez na adolescência; Adolescente; Recém-nascido; Vulnerabilidade social; Serviços de saúde do adolescente

Abstract: Objective: to compare obstetric and neonatal outcomes among adolescent and adult mothers. Method: cross-sectional study based on data from the Sistema de Informações de Nascidos Vivos (Live Birth Information System) comparing socio-demographic, obstetric, and neonatal variables of adolescent and adult mothers from Rio

\footnotetext{
${ }^{\text {I }}$ Enfermeira. Mestranda em Saúde Coletiva. Universidade do Vale do Rio dos Sinos, São Leopoldo, RS, Brasil. E-mail: betinaberlitz@gmail.com Orcid: https://orcid.org/0000-0002-9860-5432

II Enfermeira. Doutora em Enfermagem. Docente no Departamento de Assistência e Orientação Profissional da Escola de Enfermagem da Universidade Federal do Rio Grande do Sul. Porto Alegre, RS, Brasil. E-mail: carlise.nora@ufrgs.br Orcid: https://orcid.org/0000-0001-5501-2146

III Enfermeira. Doutora em Enfermagem. Professora dos Programas de Pós-Graduação em Enfermagem e Saúde Coletiva da Universidade do Vale do Rio dos Sinos, São Leopoldo, RS, Brasil. E-mail: rafaschaefer@unisinos.br Orcid: https://orcid.org/0000-0002-1484-8067

IV Enfermeira. Doutora em Gerontologia Biomédica. Professora Associada, Departamento de Enfermagem, Universidade Federal de Ciências da Saúde de Porto Alegre, Porto Alegre, RS, Brasil. E-mail: karinv@ufcspa.edu.br Orcid: https://orcid.org/0000-0002-2546-9281

V Estatística. Doutora em Saúde da Criança e do Adolescente. Analista Pesquisadora do Departamento de Economia e Estatística da SEPLAG, Porto Alegre, RS, Brasil. E-mail: marilyn.agranonik@gmail.com Orcid: https://orcid.org/0000-0003-2699-9628

VI Assistente Social. Doutora em Educação. Docente Titular do Programa de Pós-Graduação em Enfermagem da Universidade do Vale do Rio dos Sinos, Porto Alegre, RS, Brasil. E-mail: barbiani@unisinos.br Orcid: https://orcid.org/0000-0002-1841-774X
} 
Grande do Sul in the period 2010-2016. The Chi-square test evaluated statistical association and the prevalence ratio was estimated. Results: there were significant differences between mothers aged 10-14 and those from other strata: prevalence of black or brown-skinned race/color ( $\mathrm{PR}=1.53$; CI95\% 1.46-1.60), inadequate prenatal care ( $P R=1.89$; IC95\% 1.84-1.94), prematurity ( $P R=1.35$; CI95\% 1.27-1.43), low birth weight ( $P R=1.34$; IC95\% 1.25-1.43) and lower performance in the 1st minute Apgar score ( $P R=5.68$; IC95\% 5.49-5.88). Conclusion: prevalence of risk factors for obstetric and neonatal outcomes of adolescents aged 10-14 requires careful health evaluation. Early pregnancy in adolescence requires the activation of interdisciplinary teams, and family, social assistance and socio-legal protection networks.

Descriptors: Pregnancy in adolescence; Adolescent; Infant, Newborn; Social vulnerability; Adolescent health services

Resumen: Objetivo: comparar los resultados obstétricos y neonatales entre las madres adolescentes y adultas. Método: estudio transversal basado en datos del Sistema de Información de Nacimientos Vivos comparando las variables sociodemográficas, obstétricas y neonatales de madres adolescentes y adultas de Rio Grande do Sul en el período 2010-2016. Se estimó la prueba de Chi-cuadrado la asociación estadística y la relación de prevalencia. Resultados: hubo diferencias significativas entre las madres de 10 a 14 años y las de los otros estratos: prevalencia de raza/color negro o marrón (RP-1,53; IC95\% 1,46-1,60), atención prenatal inadecuada (RP-1,89; IC95\% 1,84-1,94), prematuridad (RP-1.35; IC95\% 1,27-1,43), bajo peso al nacer (RP-1,34; IC95\% 1,25-1,43) y menor rendimiento en el primer minuto de Apgar (RP-5.68; IC95\% 5,49-5,88). Conclusión: la prevalencia de factores de riesgo para los resultados obstétricos y neonatales de adolescentes de 10 a 14 años requiere una evaluación cuidadosa de la salud. El embarazo precoz en la adolescencia requiere la activación de equipos interdisciplinarios y redes de familiares, de asistencia social y de protección socio-jurídica.

Descriptores: Embarazo en adolescencia; Adolescente; Recién nacido; Vulnerabilidad social; Servicios de salud del adolescente

\section{Introdução}

A adolescência, apesar de ser um período de poucos anos, carrega consigo intensas e significativas mudanças formativas em todos os níveis de desenvolvimento físico, cognitivo, social, emocional e sexual. Por essa razão, requer políticas e programas sociais específicos, que mantenham e ampliem os ganhos já obtidos em saúde da primeira infância. Tais investimentos, além de produzirem benefícios imediatos à proteção e saúde dos adolescentes, têm efeitos intergeracionais, repercutindo positivamente no percurso para suas vidas adultas e para seus filhos. ${ }^{1-2}$

Outra característica desta fase é sua heterogeneidade, considerando a natureza das mudanças e seus impactos. Assim sendo, é oportuno falar de "adolescências”, já que o período pode ser dividido em duas fases principais: a primeira, que compreende os adolescentes entre 10 
e 14 anos, configura o início do processo de adolescer e é marcada por fenômenos biológicos, pela identificação com seus pares, melhor compreensão do seu gênero e do reconhecimento de sua sexualidade; e a segunda, com adolescentes entre 15 e 19 anos e caracterizada como por busca de autonomia, independência financeira, acesso ao trabalho e ligação com a vida adulta. Essa delimitação etária também é adotada pela Organização Mundial da Saúde (OMS) e pelo Ministério da Saúde (MS) para orientar a formulação de políticas públicas. ${ }^{3-4}$ Entre os elementos comuns a esses estratos etários, destaca-se a temática da saúde sexual e reprodutiva, cujos contornos polêmicos chocam-se constantemente com a estrutura social, seus valores e condicionantes. Contudo, é um debate de fundamental importância na afirmação da vida de adolescentes e de seus direitos. ${ }^{4}$

Estudo que analisou indicadores de saúde sexual e reprodutiva de adolescentes com base nos dados das três edições da Pesquisa Nacional de Saúde do Escolar (PeNSE) revelou que a idade média de iniciação sexual dos adolescentes brasileiros é de 13 anos, e 19,5\% da amostra informaram não utilizar nenhum método de proteção. ${ }^{5}$ Contudo, a iniciação sexual traz, entre seus possíveis desfechos, a gravidez precoce. Segundo a OMS, a gravidez é considerada precoce quando a adolescente tem o primeiro filho antes dos 18 anos de idade. ${ }^{1,6} \mathrm{~A}$ despeito de que engravidar seja o desejo de muitas mulheres, inclusive de adolescentes, a gravidez precoce, dependendo das condições em que ocorre, pode acarretar significativos riscos e agravos gestacionais, obstétricos, neonatais e sociais, além de impactar o futuro de várias gerações. ${ }^{1,5,7-10}$

Estudos apontam que a gravidez na adolescência está diretamente relacionada a nascimentos de baixo peso, prematuridade e taxa maior de mortalidade infantil em mães adolescentes. ${ }^{1,9,11}$ Ademais, pela complexidade dos cuidados que demanda, a gravidez precoce produz implicações importantes para os sistemas de saúde e proteção social., ${ }^{3,12}$ Além dos riscos e prejuízos associados ao binômio mãe-filho, as repercussões sociais da gravidez precoce nas 
Fatores de risco aos desfechos obstétricos e neonatais de mães adolescentes $\mid 4$

vidas presente e futura dos jovens pais podem determinar a desistência dos estudos e a inserção precária no mercado de trabalho, especialmente em grupos populacionais vulneráveis., ${ }^{912-13}$

A taxa global da gravidez na adolescência vem diminuindo no estrato de 15 a 19 anos e mantém tendência de estabilização entre meninas de 10 a 14 anos. ${ }^{8,14}$ Trata-se de um fenômeno prevalente nos países subdesenvolvidos e em desenvolvimento. ${ }^{15}$ A América Latina e Caribe, por exemplo, possuem a segunda maior taxa de gravidez na adolescência do mundo, estimada em 66,5 nascimentos para cada 1.000 mulheres entre 15 e 19 anos. ${ }^{1}$ O Brasil apresenta panorama análogo ao da tendência mundial de redução da taxa de gravidez na adolescência. Entretanto, na faixa de 15 a 19 anos, a taxa é estimada em 68,4 nascimentos para cada 1.000 adolescentes, média superior à da América Latina e Caribe, enquanto o estrato de 10 a 14 anos, os números permanecem estáveis. ${ }^{1,6,15}$

Devido a esse preocupante cenário, o Brasil instituiu, recentemente, no âmbito legal e programático, alguns dispositivos para o enfrentamento do fenômeno. No Sistema Único de Saúde (SUS), houve a pactuação interfederativa do indicador "14", referente ao monitoramento da proporção de gravidez na adolescência entre as faixas etárias de 10 a 19 anos, que passou a ser obrigatório para todos os estados e municípios brasileiros a partir de $2017 .{ }^{16}$ Ainda em 2019, foi instituída no Estatuto da Criança e do Adolescente (ECA) a Semana Nacional de Prevenção da Gravidez na Adolescência, a ser realizada anualmente com o objetivo de disseminar informações sobre medidas preventivas e educativas que contribuam para a redução da incidência de gravidez na adolescência. ${ }^{17}$

O Rio Grande do Sul (RS) é o estado do Brasil que apresenta os menores índices de gravidez na adolescência e possui diretrizes programáticas específicas para atenção à saúde sexual e reprodutiva de adolescentes desde 2010. Apesar de apresentar uma diminuição da proporção geral de nascidos vivos de mães adolescentes desde 2013, a proporção de nascidos vivos 
de meninas de 10 a 14 anos se mantém estável no estado, o que revela um cenário semelhante ao nacional e internacional, isto é, um problema de saúde pública a ser enfrentado e superado.9-10,15

Nessa faixa etária, além dos complexos riscos gestacionais e de saúde, estudos apontam a possível associação da gravidez à violência sexual e ao casamento infantil. O fenômeno da gravidez indesejada em menores de 14 anos com interrupção negada, seja por falta de acesso ou pelo atraso no atendimento, é nomeado mundialmente de gravidez infantil forçada ou maternidade infantil. ${ }^{1,6,11,15}$ Entretanto, embora seja uma temática relevante nos âmbitos epidemiológico e social, a produção científica sobre as implicações da gravidez precoce ainda é incipiente, sendo abordada de forma agregada e/ou secundária às demais faixas etárias, sem distinções, como se fosse um período homogêneo. ${ }^{4,11}$

Em face desse contexto, formulou-se como questão de pesquisa: Quais são as diferenças entre os desfechos obstétricos e neonatais entre as mães adolescentes e adultas no estado do RS? Nessa perspectiva, este estudo tem como objetivo comparar os desfechos obstétricos e neonatais entre as mães adolescentes e mulheres adultas.

\section{Método}

Trata-se de um estudo transversal, que utilizou a base de dados do Departamento de Informática do Sistema Único de Saúde (DATASUS) a partir de informações do Sistema de Informações sobre Nascidos Vivos (SINASC), que tem como fonte a Declaração de Nascido Vivo (DNV).

A população pesquisada incluiu todas as mães de nascidos vivos no estado do RS, no período de 2010 até 2016. A série histórica justifica-se pelo ano de publicação das Diretrizes Nacionais e da implantação da Política Estadual de Atenção Integral à Saúde de Adolescentes no RS (PEAISA), 2010. Além disso, 2016 foi o último ano com dados disponíveis e confirmados. A coleta de dados ocorreu em dezembro de 2017, com atualizações que seguiram até maio de 2018. Associações entre características sociodemográficas, obstétricas, neonatais e grupos por 
Fatores de risco aos desfechos obstétricos e neonatais de mães adolescentes $\mid 6$

faixa etária foram investigadas para fins de comparação e análise. As variáveis foram coletadas por categorias de informações e grupo etário (10 a 14, 15 a 19, 20 anos ou mais):

a) sociodemográficas da mãe: idade, raça/cor e situação conjugal (com ou sem parceiro);

b) gestação, parto e nascimento: local de ocorrência (hospital ou outro), número de consultas de pré-natal (até 6 e 7 ou mais consultas), idade gestacional ( $<37$ e $\geq 37$ semanas), tipo de gravidez (única e dupla ou mais) e tipo de parto (vaginal e por cesariana);

c) recém-nascido: peso ao nascer (classificado em baixo peso ao nascer, $<2500 \mathrm{~g}$; e $\geq 2500 \mathrm{~g}$ ), Índice de Apgar no $1^{\circ}$ e no $5^{\circ}$ minuto (0-7 e 8-10) e anomalia congênita (sim e não).

Esta pesquisa segue os preceitos éticos estabelecidos na Resolução no 510 de 07 de abril de 2016, considerando que utiliza informações de acesso público, nos termos da Lei n 12.527 de 18 de novembro de 2011, dessa forma não necessitando de registro ou avaliação pelo sistema CEP/CONEP.

Os dados foram exportados para planilhas no Microsoft Excel ${ }^{\oplus}$, organizados e categorizados. As variáveis foram descritas por frequências absolutas $(\mathrm{N})$ e relativas (\%). A razão de prevalência $(\mathrm{RP})$ foi utilizada como medida de associação, com seu respectivo intervalo de 95\% de confiança (IC95\%). Para avaliar tendência, foi utilizado o teste Qui-quadrado de tendência, que avalia se a prevalência de cada grupo etário aumentou ou diminuiu com o passar dos anos. Por meio do teste Qui-Quadrado de Pearson, foi avaliada a existência de associação entre variáveis. No caso de significância estatística, utilizou-se a análise de resíduos para identificar quais categorias estavam associadas. Para todas as análises foi considerado o nível de significância $(\alpha)$ de $5 \%(\mathrm{p}<0,05)$. As análises foram realizadas no software Statistical Package for the Social Sciences (SPSS), versão 18.0.

\section{Resultados}

O estado do RS, no período de 2010 a 2016, registrou 984.296 nascidos vivos (NV), sendo que mães adolescentes (10 a 19 anos) representaram 15,53\% ( $\mathrm{N}=152.812)$ do total. A Tabela 1 
apresenta a evolução dos nascimentos, por ano e faixa etária da mãe, evidenciando um declínio no percentual de NV de mães nas faixas etárias de 10 a 14 anos e 15 a 19 anos ao longo dos anos. Por outro lado, houve aumento na proporção de NV com mães adultas.

Tabela 1 - Distribuição dos nascidos vivos por faixa etária da mãe, no período de 2010-2016, Rio Grande do Sul, Brasil, 2018

\begin{tabular}{|c|c|c|c|c|c|c|}
\hline \multicolumn{7}{|c|}{ Faixa etária materna (em anos) } \\
\hline & \multicolumn{2}{|c|}{$10-14$} & \multicolumn{2}{|c|}{ 15-19 } & \multicolumn{2}{|c|}{20 ou mais } \\
\hline Ano & $\mathbf{n}$ & $\%$ & $\mathbf{n}$ & $\%$ & $\mathbf{n}$ & $\%$ \\
\hline 2010 & 937 & 0,70 & 20.904 & 15,69 & 111.385 & 83,61 \\
\hline 2011 & 903 & 0,66 & 21.092 & 15,32 & 115.713 & 84,03 \\
\hline 2012 & 914 & 0,66 & 21.688 & 15,61 & 116.332 & 83,73 \\
\hline 2013 & 909 & 0,64 & 21.715 & 15,36 & 118.723 & 84,00 \\
\hline 2014 & 938 & 0,65 & 21.510 & 15,01 & 120.864 & 84,34 \\
\hline 2015 & 869 & 0,59 & 20.700 & 13,95 & 126.790 & 85,46 \\
\hline 2016 & 765 & 0,54 & 18.968 & 13,41 & 121.677 & 86,05 \\
\hline $\mathrm{P}^{*}$ & & $<0,001$ & & $<0,001$ & & $<0,001$ \\
\hline
\end{tabular}

Fonte: Sistema de Informação sobre Nascidos Vivos (SINASC); *Valor P para o teste Qui-quadrado de tendência.

Houve associação entre grupo etário materno e as variáveis raça/cor $(p<0,001)$, situação conjugal $(\mathrm{p}<0,001)$, local de ocorrência do parto $(\mathrm{p}<0,001)$, número de consultas de pré-natal $(\mathrm{p}<0,001)$ e tipo de parto $(\mathrm{p}<0,001)$ (Tabela 2$)$.

Tabela 2 - Características sociodemográficas, da gestação e do parto segundo faixa etária da mãe, no período de 2010-2016, Rio Grande do Sul, Brasil, 2018

\begin{tabular}{|c|c|c|c|c|c|c|c|}
\hline \multirow[b]{3}{*}{ Variável } & \multicolumn{4}{|c|}{ Faixa etária materna (em anos) } & \multirow{2}{*}{\multicolumn{2}{|c|}{20 ou mais }} & \multirow[b]{3}{*}{ Valor $\mathbf{p}^{*}$} \\
\hline & \multicolumn{2}{|c|}{ 10-14 } & \multicolumn{2}{|c|}{ 15-19 } & & & \\
\hline & $\mathbf{n}$ & $\%$ & $\mathbf{n}$ & $\%$ & $\mathbf{n}$ & $\%$ & \\
\hline Raça/Cor & & & & & & & $<0,001$ \\
\hline Branca & 4.694 & 78,0 & 114.283 & 79,3 & 703.472 & 85,6 & \\
\hline Preta e Parda & 1.322 & 22,0 & 29.798 & 20,7 & 118.052 & 14,4 & \\
\hline Situação conjugal & & & & & & & $<0,001$ \\
\hline Com parceiro & 714 & 11,5 & 34.617 & 23,8 & 420.502 & 50,8 & \\
\hline Sem parceiro & 5.490 & 88,5 & 111.090 & 76,2 & 406.446 & 49,2 & \\
\hline Local de Ocorrência & & & & & & & $<0,001$ \\
\hline Hospital & 6.192 & 99,3 & 146.138 & 99,7 & 828.693 & 99,7 & \\
\hline Outros & 43 & 0,7 & 435 & 0,30 & 2.773 & 0,33 & \\
\hline
\end{tabular}


Fatores de risco aos desfechos obstétricos e neonatais de mães adolescentes $\mid 8$

№ de consultas de pré-

$<0,001$

natal

$<7$ consultas

2.901

$46,8 \quad 56.560$

$38,8 \quad 204.619$

24,7

$\geq 7$ consultas

3.296

53,2

89.175

61,2

622.894

75,3

Tipo de Parto

Vaginal

3.484

$55,9 \quad 79.616$

$54,3 \quad 297.654$

$<0,001$

Por cesariana

2750

44,

66.912

$45,7 \quad 533.630$

35,8

Fonte: Sistema de Informação sobre Nascidos Vivos (SINASC); A análise das raças/cores amarela, indígena e a categoria "ignorado" foram suprimidas devido ao baixo valor para a avaliação estatística. Dados apresentados como n (\%). Valores totais podem diferir devido a informações em branco ou ignoradas. *Valor p para o teste Qui-Quadrado.

A avaliação das características sociodemográficas maternas mostra maiores prevalências de mulheres de raças/cores preta e parda e sem parceiro quanto menor a faixa etária materna (Tabela 3). Apesar do local predominante de ocorrência do parto ser o hospital, a prevalência é duas vezes maior de o parto ocorrer em outros locais para as adolescentes de 10 a 14 anos, quando comparadas às mulheres adultas (RP=2,07; IC95\%: 1,53-2,79).

Em relação às consultas de pré-natal, verificou-se que, quanto menor a idade materna, menor probabilidade de a mulher ter realizado mais de 7 consultas ( $\mathrm{RP}=1,89$; IC95\%: $1,84-1,94$ para as adolescentes mais jovens e RP=1,57; IC95\%: 1,56-1,58 para o grupo de 15 a 19 anos). Quanto ao tipo de parto, os estratos etários adolescentes registraram maiores percentuais de parto vaginal, quando comparados às mulheres adultas ( $\mathrm{RP}=1,56$; IC95\%: 1,53-1,60 para as adolescentes mais jovens e RP=1,52; IC95\%: 1,51-1,53 para as de 15 a 19 anos) (Tabela 3).

Tabela 3 - Razão de prevalência para características maternas da gestação e do parto, comparando adolescentes de 10 a 14 anos e de 15 a 19 anos com mulheres adultas, no período de 2010-2016, Rio Grande do Sul, Brasil, 2018

\begin{tabular}{|c|c|c|c|c|c|c|}
\hline \multirow{3}{*}{$\begin{array}{l}\text { Desfecho } \\
\text { Raça/cor preta ou parda }\end{array}$} & \multicolumn{3}{|c|}{ Adolescentes de 10-14 anos } & \multicolumn{3}{|c|}{ Adolescentes de 15-19 anos } \\
\hline & \multirow{2}{*}{$\begin{array}{r}\mathbf{R P} \\
1,53\end{array}$} & \multicolumn{2}{|c|}{ IC95\% } & \multirow{2}{*}{$\begin{array}{l}\mathbf{R P} \\
1,44\end{array}$} & \multicolumn{2}{|c|}{ IC95\% } \\
\hline & & 1,46 & 1,60 & & 1,42 & 1,46 \\
\hline Sem parceiro & 1,80 & 1,78 & 1,82 & 1,55 & 1,54 & 1,56 \\
\hline Ocorrência do parto fora do hospital & 2,07 & 1,53 & 2,79 & 0,89 & 0,80 & 0,98 \\
\hline № de consultas de pré-natal $<7$ & 1,89 & 1,84 & 1,94 & 1,57 & 1,56 & 1,58 \\
\hline Parto vaginal & 1,56 & 1,53 & 1,60 & 1,52 & 1,51 & 1,53 \\
\hline
\end{tabular}

Legenda: RP - razão de prevalência, IC95\% - intervalo de 95\% de confiança. 
A análise dos desfechos obstétricos (Tabela 4) mostrou maiores prevalências de baixo peso ao nascer para os dois grupos de mães adolescentes, quando comparadas às mães de 20 anos ou mais $(\mathrm{p}<0,001)$. Recém-nascidos de mães adolescentes apresentaram maior prevalência de Índice de Apgar inferior a 7 pontos no $1^{\circ}$ e no $5^{\circ}$ minuto, em comparação às mães adultas $(\mathrm{p}<0,001)$.

Tabela 4 - Características do recém-nascido segundo faixa etária da mãe, no período de 20102016, Rio Grande do Sul, Brasil, 2018

\begin{tabular}{|c|c|c|c|c|c|c|c|}
\hline \multirow[b]{3}{*}{ Variável } & \multicolumn{6}{|c|}{ Faixa etária materna (em anos) } & \multirow[b]{3}{*}{ Valor $\mathbf{P}^{*}$} \\
\hline & \multicolumn{2}{|c|}{$10-14$} & \multicolumn{2}{|c|}{$15-19$} & \multicolumn{2}{|c|}{20 ou mais } & \\
\hline & $\mathbf{n}$ & $\%$ & $\mathbf{n}$ & $\%$ & $\mathbf{n}$ & $\%$ & \\
\hline Idade Gestacional & & & & & & & $<0,001$ \\
\hline$<37$ semanas & 947 & 15,5 & 16.484 & 11,4 & 94.504 & 11,5 & \\
\hline$\geq 37$ semanas & 5.159 & 84,5 & 128.136 & 88,6 & 727.753 & 88,5 & \\
\hline Peso ao nascer & & & & & & & $<0,001$ \\
\hline$<2500 \mathrm{~g}$ & 774 & 12,4 & 13.948 & 9,5 & 77.270 & 9,3 & \\
\hline$\geq 2500 \mathrm{~g}$ & 5.460 & 87,6 & 132.616 & 90,5 & 754.128 & 90,7 & \\
\hline Apgar $1^{\circ} \mathrm{min}$ & & & & & & & $<0,001$ \\
\hline 0 a 7 & 1.073 & 68,0 & 21.139 & 14,6 & 98.597 & 12,0 & \\
\hline 8 a 10 & 505 & 32,0 & 123.581 & 85,4 & 724.694 & 88,0 & \\
\hline Apgar $5^{\circ} \mathrm{min}$ & & & & & & & $<0,001$ \\
\hline 0 a 7 & 248 & 4,0 & 4.125 & 2,8 & 18.432 & 2,2 & \\
\hline 8 a 10 & 5.883 & 96,0 & 140.661 & 97,2 & 805.210 & 97,8 & \\
\hline Anomalia & & & & & & & $<0,001$ \\
\hline Congênita & & & & & & & \\
\hline Sim & 71 & 1,2 & 1.432 & 1,0 & 7.609 & 0,9 & \\
\hline Não & 6.050 & 98,8 & 142.268 & 99,0 & 809.640 & 99,1 & \\
\hline
\end{tabular}

Fonte: Sistema de Informação sobre Nascidos Vivos (SINASC); A análise das raças/cores amarela, indígena e a categoria "ignorado" foram suprimidas devido ao baixo valor para a avaliação estatística. Valores totais podem diferir devido a informações em branco ou ignoradas. *Valor p para o teste Qui-Quadrado.

A prevalência de prematuridade foi significativamente maior entre os recém-nascidos com mães de 10 a 14 anos (RP=1,35; IC95\%: 1,27-1,43), em comparação às mães adultas (Tabela 5). Não houve diferença na prevalência de prematuridade entre filhos de mulheres com 15 a 19 anos e de mulheres adultas ( $\mathrm{RP}=0,99$; IC95\%: 0,98-1,01). Entretanto, a prevalência de anomalias congênitas foi significativamente maior apenas para os recém-nascidos de mães de 15 a 19 anos, 
quando comparados aos recém-nascidos de mulheres com 20 anos ou mais (RP=1,07; IC95\%: 1,01-1,13) (Tabela 5).

Tabela 5 - Razão de prevalência para os desfechos neonatais, comparando mães adolescentes de 10 a 14 anos e de 15 a 19 anos a mulheres adultas, no período de 2010-2016, Rio Grande do Sul, Brasil, 2018

\begin{tabular}{|c|c|c|c|c|c|c|}
\hline \multirow{3}{*}{$\begin{array}{l}\text { Desfecho } \\
\text { Pré-termo }\end{array}$} & \multicolumn{3}{|c|}{ Adolescentes de 10-14 anos } & \multicolumn{3}{|c|}{ Adolescentes de 15-19 anos } \\
\hline & \multirow{2}{*}{$\begin{array}{l}\mathbf{R P} \\
1,35\end{array}$} & \multicolumn{2}{|c|}{ IC95\% } & \multirow{2}{*}{$\begin{array}{l}\mathbf{R P} \\
0,99\end{array}$} & \multicolumn{2}{|c|}{ IC95\% } \\
\hline & & 1,27 & 1,43 & & 0,98 & 1,01 \\
\hline Peso ao nascer $<2500 \mathrm{~g}$ & 1,34 & 1,25 & 1,43 & 1,02 & 1,01 & 1,04 \\
\hline Apgar $1^{\circ}$ min de 0 a 7 & 5,68 & 5,49 & 5,88 & 1,22 & 1,20 & 1,24 \\
\hline Apgar $5^{\circ}$ min de 0 a 7 & 1,81 & 1,60 & 2,04 & 1,27 & 1,23 & 1,32 \\
\hline Presença de anomalia congênita & 1,25 & 0,99 & 1,57 & 1,07 & 1,01 & 1,13 \\
\hline
\end{tabular}

Legenda: RP - razão de prevalência, IC95\% - intervalo de 95\% de confiança.

\section{Discussão}

O estudo apontou que, no estado do RS, na série histórica de 2010 a 2016, a proporção de nascimentos diminuiu nos dois estratos etários de mães adolescentes, e aumentou no estrato de 20 anos ou mais. O percentual de nascimentos acompanha a tendência internacional e nacional de queda. No entanto, o Relatório do Fundo das Nações Unidas de 2016 alerta para as evidências da desigualdade social: enquanto há redução da taxa de fertilidade nos estratos sociais mais favorecidos, prevalece a ocorrência cada vez mais precoce das gestações nas regiões menos desenvolvidas, a exemplo da América Latina. ${ }^{1}$

Estudo realizado nas cinco regiões do Brasil avaliou a tendência da gravidez nos dois segmentos etários da adolescência (10 a 14 e 15 a 19 anos) no período de 2000 a 2011, relacionando-a ao Índice de Desenvolvimento Humano (IDH). A investigação revelou que há uma relação inversamente proporcional entre o número de NV e o IDH, sendo a região Sudeste a que apresenta o menor número de NV e o maior o IDH do Brasil no período. O estudo revelou, ainda, o declínio de casos de gravidez na adolescência em todo o território nacional, mas com 
um incremento de 5\% de gravidez na faixa etária de 10 a 14 anos nas regiões Norte e Nordeste, ambas com os menores IDHs do país. ${ }^{14}$

Entre as mães adolescentes, foi observado maior prevalência de desfechos desfavoráveis aos recém-nascidos cujas mães tinham idade entre 10 e 14 anos, comparados ao segmento etário de mães entre 15 e 19 anos e adultas. Nessa mesma direção, estudo internacional aponta que os maiores ganhos em saúde materna nas últimas décadas beneficiaram mais as mulheres jovens do que as adolescentes. ${ }^{2}$

No indicador raça/cor, a autodeclaração da cor branca apresentou o maior percentual em todos os ciclos etários, o que pode ser em parte justificado pelas características étnicas do estado. ${ }^{18}$ Entretanto, houve significância estatística indicando maior percentual de mães de raça/cor negra ou parda nos estratos adolescentes. Esse achado corrobora com os resultados de outras pesquisas, podendo indicar situação de maior vulnerabilidade quando associado a outros determinantes, por exemplo, a escolaridade: estudos realizados no Brasil, tendo como fonte o SINASC, evidenciaram que mulheres adolescentes com baixa escolaridade e da raça/cor negra realizaram menos consultas de pré-natal, quando comparadas às mães adultas com maior escolaridade e da raça/cor branca. ${ }^{11,19}$

A maternidade, para a maioria das adolescentes, ocorreu sem o vínculo com parceiro, ou seja, sem a convivência do pai, seja biológico ou afetivo, o que é uma realidade semelhante às encontradas em outro estudo. ${ }^{11}$ No entanto, o percentual de $11,5 \%$ de mães adolescentes de 10 a 14 anos declaradas em união estável ou casadas sugere a indagação sobre as circunstâncias da gravidez e da relação de conjugalidade, considerada casamento infantil e crime sexual no Brasil. Trata-se de uma realidade preocupante, sobretudo devido às vulnerabilidades que podem estar associadas às desigualdades sociais, evidenciadas até aqui pela cor da pele e baixa escolaridade das mães adolescentes, podendo, ainda, desvelar situações de violência sexual. 
Fatores de risco aos desfechos obstétricos e neonatais de mães adolescentes | 12

O casamento infantil é reconhecido internacionalmente como uma violação dos direitos humanos, pois pode impedir a adolescente de exercer seu direito de escolher livremente sobre um cônjuge e ter informações completas sobre essa escolha. ${ }^{20}$ Ademais, altas taxas de casamento infantil estão associadas a menos planejamento familiar, maior fecundidade, gravidez indesejada, maior risco de complicações durante o parto, progresso acadêmico limitado e redução do potencial de renda. ${ }^{6,21}$ Revela-se a importância da prevenção do casamento infantil, assim como os efeitos deletérios intergeracionais causados pela gravidez precoce.

Um estudo constatou que crianças nascidas de mulheres que se casaram antes dos 18 anos, em comparação àquelas cujas mães se casaram mais tarde, têm entre $25 \%$ e $29 \%$ mais chances de comprometimento do desenvolvimento saudável. Além disso, a localização geográfica e a educação primária foram os fatores contextuais que explicaram a maior parte dessa relação. ${ }^{22}$ Assim, a prevenção do casamento infantil pode impactar, ainda, na redução de $70 \%$ nas taxas de mortalidade materna e uma diminuição de $3 \%$ nas taxas de mortalidade infantil de um país. ${ }^{21}$

O ECA é o marco fundante no reconhecimento de adolescentes como sujeitos de direitos no cenário brasileiro. Tal instrumento jurídico dispõe sobre a doutrina de proteção integral, demarca esta população como prioridade absoluta e afirma o direito à vida, saúde e proteção a qualquer forma de violência. ${ }^{23}$ Para o propósito deste estudo, cabe destacar que o ECA deve garantir todas as oportunidades para o pleno desenvolvimento de adolescentes, incluindo os direitos sexuais e reprodutivos e o acesso à educação sexual. ${ }^{4}$

Perante a legislação brasileira, toda gestação ocorrida até os 14 anos de idade é tida como crime sexual (estupro de vulnerável), independentemente da idade do autor ou consentimento da vítima, com notificação compulsória a ser realizada em até 24 horas. Contudo, mesmo com o rigor da Lei, estudo de abrangência nacional apontou que, dos $31.611 \mathrm{NV}$ de mães com até 13 anos de idade, somente $4 \%$ destes foram notificados no Sistema de Informação de Agravos de Notificação (SINAN). ${ }^{11}$ 
Em relação aos indicadores envolvendo o ciclo gravídico-puerperal, o estudo revelou que, quanto mais jovens as mães, maior o risco de realizarem um pré-natal inadequado, considerando que o MS classifica como pré-natal adequado a realização de no mínimo sete consultas. Essa realidade converge com outros estudos realizados no país, que demonstram que, quanto mais avançada a idade da mãe, mais adequado é o pré-natal.9,19,24-25

As demais variáveis analisadas (peso ao nascer, idade gestacional, tipo de parto e Índice de Apgar) demonstraram resultados preocupantes nos estratos etários adolescentes, principalmente no estrato de 10 a 14 anos. Estudo do Reino Unido ${ }^{26}$ corrobora com os resultados ao indicar que a probabilidade de muito baixo peso ao nascer $(<1000 \mathrm{~g})$ foi significativamente maior no grupo de adolescentes ( $\leq 19$ anos), em comparação com o grupo de mulheres de 20 a 34 anos. O peso inferior a $2500 \mathrm{~g}$ e idade gestacional menor que 37 semanas, quando associados, são considerados maiores fatores de risco para mortalidade neonatal. Ademais, estudo realizado em uma maternidade pública na Amazônia revelou taxas maiores de prematuridade e baixo peso ao nascer em adolescentes, com desempenhos inferiores no recorte de 10 a 14 anos de idade. ${ }^{27}$

O maior percentual de parto vaginal foi identificado nas adolescentes entre 10 e 14 anos (55,9\%), único indicador em que o desempenho desta faixa etária é superior ao das demais. Estudo da Romênia identificou que as mães adolescentes $(<20$ anos de idade) tiveram mais probabilidade do que as mães adultas ( $>20$ a 24 anos) de darem à luz por parto vaginal, e a taxa de parto por cesariana foi menor naquele grupo. ${ }^{28}$ Ainda, outro estudo refere que "adolescentes jovens”, de 11 a 14 anos, apresentaram menor risco de parto por cesariana em comparação com adultas jovens de 20 a 24 anos. $^{29}$

Os resultados evidenciam que as mulheres dos estratos adolescentes apresentam significativa vulnerabilidade social e maior risco obstétrico e neonatal, principalmente aquelas do estrato etário de 10 a 14 anos. Por isso, a saúde sexual e reprodutiva de mulheres menores de 15 anos é reconhecida como uma prioridade e a gravidez como um fator de risco, enfatizando-se 
a necessidade de uma atenção diferenciada, que aborde as especificidades desse grupo etário, além de ações educativas e preventivas. ${ }^{9-10,30}$

Nessa perspectiva, estudos internacionais sobre a efetividade da intervenção em saúde estão sendo desenvolvidos, cujos resultados vêm apontando para uma educação sexual abrangente, envolvendo vários atores e espaços. ${ }^{1,20-21} \mathrm{O}$ investimento em conhecimento e atitudes foi apontado como evidência de alta qualidade de benefício moderado para todos os países, independentemente da renda. Tanto as intervenções lideradas por adultos quanto as por pares mostraram benefícios para comportamentos de sexo seguro, e para a prevenção da gravidez identificou-se como evidência de alta qualidade a combinação entre educação e acesso a contraceptivos. ${ }^{2}$

Vale ressaltar as limitações dos estudos realizados com dados secundários, minimizadas nesta pesquisa pela comparação das características de gravidez e nascidos vivos entre as adolescentes e mulheres adultas. Salienta-se que os estudos transversais representam a etapa inicial do processo de vigilância em saúde e, neste caso, foram revelados elementos importantes para que estudos subsequentes investiguem as circunstâncias de gravidez, sobretudo em situação de precocidade, e a interface com a violência sexual.

\section{Conclusão}

Neste estudo, o conjunto das características sociodemográficas e os desfechos gestacionais, obstétricos e neonatais das mães adolescentes demonstraram piores resultados, a saber, prematuridade, baixo peso ao nascer e menor cobertura pré-natal, quando comparados com os resultados de mães adultas. Ainda, o risco foi significativamente maior no estrato etário de 10 a 14 anos.

Os indicadores apontam para as vulnerabilidades que cercam a adolescência, no âmbito dos direitos sexuais e reprodutivos. Nesse sentido, é fundamental reconhecer os/as adolescentes como indivíduos de direitos e garantir seu acesso à educação sexual, aos serviços e aos insumos de saúde, rompendo com imposições morais e entraves no atendimento. Faz-se necessária uma 
superação do discurso alarmista sobre a gravidez na adolescência, construindo e reconstruindo caminhos de educação em saúde para o diálogo sobre o exercício sexual e reprodutivo seguro, indo ao encontro de projetos de vida, nesta fase tão significativa da vida humana. Além disso, é preciso efetivar o acesso de adolescentes e de suas redes de apoio aos territórios de saúde, a fim de que seus direitos sejam reconhecidos e garantidos.

Gestantes e mães adolescentes, a considerar o primeiro recorte etário situado entre 10 e 14 anos, supõem acionamentos do setor de Saúde e de Proteção. Além das repercussões clínicas e psicológicas, há implicações na ordem da proteção social envolvidas nas gestações precoces, pois há possibilidade de resultarem de violência sexual. Nesses casos, o atendimento da saúde não pode se restringir ao encaminhamento ao pré-natal nem à puericultura ao recém-nascido. Por isso, ressalta-se a importância de um olhar diferenciado às gestantes adolescentes precoces, com uma avaliação criteriosa da equipe de saúde, pois pode-se revelar um cenário de violação de direitos em suas mais diversas expressões, sendo indispensável o acionamento de equipes interdisciplinares, redes familiares, socioassistenciais e de proteção sociojurídica.

\section{Referências}

1. Pan American Health Organization (OPAS); World Health Organization (WHO). Accelerating progress toward the reduction of adolescent pregnancy in Latin America and the Caribbean [Internet]. Washington (DC); 2016 [cited 2019 Jun 20]. Available from: http://iris.paho.org/xmlui/bitstream/handle/123456789/34493/9789275119761eng.pdf?sequence $=1 \&$ isAllowed $=y$

2. Patton GC, Sawyer SM, Santelli JS, Ross DA, Afifi R, Allen NB, et al. Our future: a Lancet commission on adolescent health and wellbeing. The Lancet. 2016;387(10036):2423-78. doi: 10.1016/S01406736(16)00579-1

3. Ministério da Saúde (BR), Secretaria de Atenção à Saúde, Departamento de Ações Programáticas e Estratégicas. Proteger e cuidar da saúde de adolescentes na atenção básica. Brasília (DF): Ministério da Saúde; 2017 [acesso em 2020 em jan 20]. Disponível em: http://bvsms.saude.gov.br/bvs/publicacoes/proteger_cuidar_adolescentes_atencao_basica.pdf

4. Moraes SP, Vitalle MSS. Direitos sexuais e reprodutivos na adolescência. Rev Assoc Med Bras. 
Fatores de risco aos desfechos obstétricos e neonatais de mães adolescentes | 16

2012;58(1):48-52. doi: 10.1590/S0104-42302012000100014

5. Felisbino-Mendes MS, Paula TF, Machado IE, Oliveira-Campos M, Malta DC. Análise dos indicadores de saúde sexual e reprodutiva de adolescentes brasileiros, 2009, 2012 e 2015. Rev Bras Epidemiol. 2018;21(Suppl 1):e180013.supl1. doi: 10.1590/1980-549720180013.supl.1

6. Wodon Q, Tavares PMT, Male C, Loureiro A. Casamento na infância e adolescência: a educação das meninas e a legislação brasileira. Washington (DC): The World Bank; 2019 [acesso em 10 jul 2020]. Disponível em: https://documents.worldbank.org/en/publication/documentsreports/documentdetail/657391558537190232/child-marriage-girls-education-and-the-law-in-brazil

7. Maranhão TA, Gomes KRO, Oliveira DC, Moita Neto JM. Repercussão da iniciação sexual na vida sexual e reprodutiva de jovens de capital do Nordeste brasileiro. Ciênc Saúde Colet. 2017;22(12):4083-94. doi: 10.1590/1413-812320172212.16232015

8. Monteiro DLM, Martins JAFS, Rodrigues NCP, Miranda FRD, Lacerda IMS, Souza FM, et al. Adolescent pregnancy trends in the last decade. Rev Assoc Med Bras. 2019;65(9):1209-15. doi: 10.1590/1806-9282.65.9.1209

9. Instituto dos Direitos da Criança e do Adolescente (INDICA); Fundo de População das Nações Unidas (UNFPA); Fundo das Nações Unidas para a Infância (UNICEF). Gravidez na adolescência no Brasil: vozes de meninas e de especialistas. Brasília (DF): INDICA; 2017 [acesso em 2020 maio 29]. Disponível em: http://unfpa.org.br/Arquivos/br_gravidez_adolescencia_2017.pdf

10. Sociedade Brasileira de Pediatria (SBP), Departamento Científico de Adolescência. Prevenção da gravidez na adolescência no Brasil: guia prático de atualização [Internet]. 2019 [acesso em 2020 maio 25];11:1-9. Disponível em: https://www.sbp.com.br/fileadmin/user_upload/Adolescencia_-_21621c-GPA__Prevencao_Gravidez_Adolescencia.pdf

11. Souto RMCV, Porto DL, Pinto IV, Vidotti CCF, Barufaldi LA, Freitas MG, et al. Estupro e gravidez de meninas de até 13 anos no Brasil: características e implicações na saúde gestacional, parto e nascimento. Ciênc Saúde Coletiva. 2017;22(9):2909-18. doi: 10.1590/1413-81232017229.13312017

12. Organização Pan-Americana da Saúde (OPAS); Organização Mundial da Saúde (OMS). Ação Global Acelerada para a Saúde de Adolescentes (AA-HA!): guia de orientação para apoiar a implementação pelos países [Internet]. Washington (DC): Organização Pan-Americana da Saúde; 2018 [acesso em 2020 fev 14]. Disponível em: https://iris.paho.org/bitstream/handle/10665.2/49095/9789275719985por.pdf? sequence $=5 \&$ is Allowed $=y$

13. Rodrigues LS, Silva MVO, Gomes MAV. Gravidez na adolescência: suas implicações na adolescência, na família e na escola. Rev Educ Emancip. 2019 maio-ago;12(2). doi: 10.18764/2358-4319.v12n2p228-252

14. Vaz RF, Monteiro DLM, Rodrigues NCP. Trends of teenage pregnancy in Brazil, 2000-2011. Rev Assoc Med Bras. 2016;62(4):330-5. doi: 10.1590/1806-9282.62.04.330 
15. Fondo de Población de las Naciones Unidas (UNFPA). 165 millones de razones: un llamado a la acción para la inversión en adolescencia y juventud en América Latina y el Caribe. UNFPA; 2019 [acceso 2020 jul 10]. Disponible en: https://brazil.unfpa.org/sites/default/files/pub-pdf/165M_ESP_WEB.pdf

16. RIO GRANDE DO SUL (Estado). Secretaria da Saúde. Resolução no 031/17 - CIB/RS. Comissão Intergestores Bipartite/RS. Porto Alegre: Secretaria da Saúde; 2017. Disponível em: http://www.ses.rs.gov.br/upload/arquivos/carga20170824/23162411-resolucao-031-17.pdf. Acesso em: 02 ago. 2020.

17. BRASIL. Lei no 13.798 , de 3 de janeiro de 2019. Acrescenta art. $8^{\circ}$-A à Lei no 8.069 , de 13 de julho de 1990, para instituir a Semana Nacional de Prevenção da Gravidez na Adolescência. Brasília, DF, 2019. Diário Oficial da União, Seção 1, p. 3. 04 jan. 2019.

18. Instituto Brasileiro de Geografia e Estatísticas (IBGE). Censo Demográfico. Rio de Janeiro: IBGE; 2010.

19. Souza ML, Lynn FA, Johnston L, Tavares ECT, Brüggemann OM, Botelho LJ. Taxa de fertilidade e desfecho perinatal em gravidez na adolescência: estudo retrospectivo populacional. Rev Latinoam Enferm. 2017;25:e2876. doi: 10.1590/1518-8345.1820.2876

20. Santelli JS, Spindler E, Moore E, McGovern T. Criminalising sexuality or preventing child marriage: legal interventions and girls' empowerment. Lancet Child Adolesc Health. 2018;3(4):206-8. doi: 10.1016/S2352-4642(18)30370-5

21. United Nations (UN), Every Woman Every Child. The global strategy for women's children's and adolescent's health (2016-2030): survive, thrive, transform [Internet]. 2015 [cited 2019 Aug 01]. Available from: https://www.everywomaneverychild.org/global-strategy/

22. Efevbera Y, Bhabha J, Farmer PE, Fink G. Girl child marriage as a risk factor for early childhood development and stunting. Soc Sci Med. 2017;185:91-101. doi: 10.1016/j.socscimed.2017.05.027

23. BRASIL. Lei no 8.069, de 13 de julho de 1990. Dispõe sobre o Estatuto da Criança e do Adolescente e dá outras providências. Diário Oficial da União: seção 1, Brasília, DF, ano 128, n. 135, p. 1-15, 16 jul. 1990. Disponível em: http://www.planalto.gov.br/ccivil_03/leis/18069.htm. Acesso em: 2 ago. 2019.

24. Fonseca SC, Monteiro DSA, Pereira CMSC, Scoralick ACD, Jorge MG, Rozario S. Desigualdades no pré-natal em cidade do Sudeste do Brasil. Ciênc Saúde Colet. 2014;19(7):1991-8. doi: 10.1590/141381232014197.04212013

25. Santos NLB, Guimarães DA, Gama CAP. A percepção de mães adolescentes sobre seu processo de gravidez. Rev Psicol Saúde. 2016;8(2):83-96. doi: 10.20435/2177-093X-2016-v8-n2(07)

26. Marvin-Dowle K, Kilner K, Burley VJ, Soltani H. Impact of adolescent age on maternal and neonatal outcomes in the Born in Bradford cohort. BMJ Open. 2018;8:e016258. doi: 10.1136/bmjopen-2017-016258 
27. Silva NND, Chaves LN, Chaves LN, Rêgo AD, Araújo DB. Análise de partos em adolescentes e repercussões perinatais em uma maternidade pública na Amazônia. Adolesc Saude [Internet]. 2018 [acesso em 2019 jun 13];15(1):50-7. Disponível em: http://www.adolescenciaesaude.com/detalhe_artigo.asp?id=708

28. Socolov DG, Iorga M, Carauleanu A, Ilea C, Blidaru I, Boiculese L, et al. Pregnancy during adolescence and associated risks: an 8-year hospital-based cohort study (2007-2014) in Romania, the country with the highest rate of teenage pregnancy in Europe. BioMed Res Int. 2017;2017(9205016):1-8. doi: $10.1155 / 2017 / 9205016$

29. Torvie AJ, Callegari LS, Schiff MA, Debiec KE. Labor and delivery outcomes among young adolescents. Am J Obstet Gynecol. 2015;213(1):95.E1-8. doi: 10.1016/j.ajog.2015.04.024

30. Fundo de População das Nações Unidas (UNFPA). Situação da população mundial 2017: mundos distantes: saúde e direitos reprodutivos em uma era de desigualdade. Brasília (DF): UNFPA; 2017 [acesso em 2019 ago 13]. Disponível em: https://brazil.unfpa.org/sites/default/files/pub-pdf/PT_WEB-SWOP2017Report.pdf

Editor Científico Chefe: Cristiane Cardoso de Paula

Editor Científico: Tânia Solange Bosi de Souza Magnago

\section{Autor correspondente}

Betina Berlitz

E-mail: betinaberlitz@gmail.com

Endereço: Programa de Pós-Graduação em Saúde Coletiva, Universidade do Vale do Rio dos Sinos. Av. Unisinos, 950 - Cristo Rei, São Leopoldo - RS, Brasil

CEP: $93022-750$

\section{Contribuições de autoria:}

\section{1 - Betina Berlitz}

Concepção e planejamento de pesquisa, coleta de dados, análise, interpretação dos dados e redação.

\section{2 - Carlise Rigon Dalla Nora}

Revisão final com participação crítica e intelectual no manuscrito.

\section{3 - Rafaela Schaefer}

Análise e interpretação dos dados e revisão final com participação crítica e intelectual no manuscrito.

\section{4 - Karin Viegas}

Planejamento metodológico e redação com participação crítica e intelectual do manuscrito. 
19 | Berlitz B, Nora CRD, Schaefer R, Viegas K, Agranonik M, Barbiani R

\section{5 - Marilyn Agranonik}

Análise e interpretação dos dados e revisão final com participação crítica e intelectual no manuscrito.

\section{6 - Rosangela Barbiani}

Concepção, planejamento e orientação do projeto de pesquisa, análise e interpretação dos dados, redação e revisão crítica.

\section{Como citar este artigo}

Berlitz B, Nora CRD, Schaefer R, Viegas K, Agranonik M, Barbiani R. Fatores de risco aos desfechos obstétricos e neonatais de mães adolescents. Rev. Enferm. UFSM. 2020 [Acesso em: Anos Mês Dia]; vol.10 e89: 1-19. DOI:https://doi.org/10.5902/2179769240813 